\title{
MEASURABLE REPRESENTATIONS OF PREFERENCE ORDERS
}

\author{
BY \\ R. DANIEL MAULDIN ${ }^{1}$
}

\begin{abstract}
A continuous preference order on a topological space $Y$ is a binary relation $\preccurlyeq$ which is reflexive, transitive and complete and such that for each $x,\{y$ : $x \preccurlyeq y\}$ and $\{y: y \leqslant x\}$ are closed. Let $T$ and $X$ be complete separable metric spaces. For each $t$ in $T$, let $B_{t}$ be a nonempty subset of $X$, let $\preccurlyeq_{t}$ be a continuous preference order on $B_{t}$ and suppose $E=\left\{(t, x, y): x \preccurlyeq_{t} y\right\}$ is a Borel set. Let $B=\{(t, x)$ : $\left.x \in B_{t}\right\}$.

THEOREM 1. There is an $\mathcal{S}(T) \otimes \mathscr{B}(X)$-measurable map $g$ from $B$ into $R$ so that for each $t, g(t, \cdot)$ is a continuous map of $B_{t}$ into $R$ and $g(t, x) \leqslant g(t, y)$ if and only if $x \preccurlyeq, y$. (Here $\delta(T)$ forms the $C$-sets of Selivanouskii and $\oiint(X)$ is a Borel field on $X$.)

THEOREM 2. If for each $t, B_{t}$ is a $\sigma$-compact subset of $Y$, then the map $g$ of the preceding theorem may be chosen to be Borel measurable.

The following improvement of a theorem of Wesley is proved using classical methods.

THEOREM 3. Let $g$ be the map constructed in Theorem 1 . If $\mu$ is a probability measure defined on the Borel subsets of $T$, then there is a Borel set $N$ such that $\mu(N)=0$ and such that the restriction of $g$ to $B \cap((T-N) \times X)$ is Borel measurable.
\end{abstract}

1. Introduction. A preference order is a binary relation $\preccurlyeq$ on a set $Y$ which is transitive, reflexive and complete. Such a relation is also known as a linear preorder. One may partition the set $Y$ into equivalence classes via the equivalence relation $x \sim y$ if and only if $x \leqslant y$ and $y \leqslant x$ (one may think of $x$ being equivalent to $y$ as meaning that $x$ and $y$ are equally "preferred"). The natural relation induced by $\preccurlyeq$ on the set of equivalence classes is a linear order. A representation or Paretian utility function of a preference order is a map $f$ from $Y$ into the real numbers such that $x \leqslant y$ if and only if $f(x) \leqslant f(y)$. One of the problems which has been of interest to mathematicians and economists concerns continuous representations of a preference order when the space $Y$ has some underlying topological structure to which the preference order is related [5, 12]. More generally, one may have parametrized versions of this problem. One may consider the space $Y$ and the preference order $\preccurlyeq$ as not being fixed, but the functions of some parameter (say time or "traders"). One may then be concerned with the problem of obtaining (continuous) representations which vary in some reasonable sense with the parameter [16]. This

Received by the editors August 29, 1980 and, in revised form, February 8, 1982.

AMS (MOS) subject classifications (1970). Primary 90A10, 54H05, 04A15, 28A20.

Key words and phrases. Preference order, continuous order preserving map, universally measurable, analytic set.

'Research supported by National Science Foundation grant MCS-81-01581 and by a Faculty Research Grant from North Texas State University. 
type of situation is known as "preference orders in markets with a continuum of traders". A discussion and references may be found in [7].

The purposes of this paper are to prove some general representation theorems and to formulate some of the unsolved problems. Some known results will be reproved either for the deeper purposes of this paper or to show these earlier results may be obtained from standard mathematical procedures and constructions without the use of forcing methods from mathematical logic.

Our setting which will be called the "fundamental hypothesis" throughout the paper is the following:

Let $T$ and $X$ be Polish (= complete separable metric) spaces. For each $t$ in $T$, let $B_{t}$ be a nonempty subset of $X$ and $\preccurlyeq_{t}$ a preference order on $B_{t}$ such that if $x \in B_{t}$, then both $\left\{y: x \preccurlyeq_{t} y\right\}$ and $\left\{y: y \preccurlyeq_{t} x\right\}$ are closed with respect to $B_{t}$. We will also assume that this entire process is "describable" or computable from some parameter. Our assumption in this direction is that

$$
E \equiv\left\{(t, x, y) \in T \times X \times X: x \preccurlyeq_{t} y\right\}
$$

is a Borel measurable subset of $T \times X \times X$.

In the context of this fundamental hypothesis we will set

$$
B=\left\{(t, x) \in T \times X: x \in B_{t}\right\} .
$$

Let us note at the outset that $B$ is a Borel measurable subset of $T \times X$ since $B=\pi_{12}(E \cap \Delta)$, where $\Delta=\{(t, x, x): t \in T$ and $x \in X\}$ and $\pi_{12}$ is the canonical projection map of $T \times X \times X$ into the first two coordinate spaces. Of course $\pi_{12}$ restricted to the Borel set $E \cap \Delta$ is a one-to-one continuous map, so the image of $E \cap \Delta$ under $\pi_{12}$ is also a Borel set [9, p. 487].

Let us make the following terminology. If $\preccurlyeq$ is a preference order on a topological space $Y$, then $\preccurlyeq$ will be said to be a continuous preference order provided that for each $x$ in $Y$, both $\{y: y \preccurlyeq x\}$ and $\{y: x \preccurlyeq y\}$ are closed. This definition is reasonable since in order for there to be a continuous representation of $\preccurlyeq$ it is, of course, necessary that these sets be closed. It is a corollary of a result of Debreu [5] that for each $t$, there is a continuous representation of $\preccurlyeq_{t}$.

Thus, one question which naturally arises is how these representations can be "pasted together". Heuristically, one could speculate that since the preference orders are "describable", there should be some reasonable manner of putting the individual representations together. Specifically:

Problem. Is there a Borel measurable map $f$ from $B$ into $R$ so that for each $t$ in $T$, $f(t, \cdot)$ is a continuous representation of $\preccurlyeq_{t}$ ?

The answer to this problem is surely negative although we do not have an example. We do prove that the answer is yes if one makes some additional assumptions concerning the $T$-sections of $B$.

THEOREM 4.3. Assume the fundamental hypothesis. If for each $t, B_{t}$ is a $\sigma$-compact subset of $X$, then there is a Borel measurable map fof $B$ into $R$ such that for each $t$ in $T$, $f(t, \cdot)$ is a continuous representation of $\preccurlyeq_{t}$.

We also show that one can always obtain a universally measurable map $f$ with no additional assumption. 
THEOREM 3.4. Assume the fundamental hypothesis. Then there is a map $g$ of $B$ into $R$ which is $\delta(T) \otimes \Re(X)$-measurable and such that for each $t, g(t, \cdot)$ is a continuous representation of $\preccurlyeq_{t}$.

Our method of proof of Theorem 3.4 yields an improvement in a theorem of Wesley [16].

THEOREM 4.1. Assume the fundamental hypothesis. There is a map $g$ of $B$ into $R$ which is $\mathcal{S}(T) \otimes \mathscr{B}(X)$-measurable such that for each $t, g(t, \cdot)$ is a continuous representation of $\preccurlyeq_{t}$ and such that if $\mu$ is a probability measure defined on the Borel subsets of $T$, then there is a Borel set $N$ such that $\mu(N)=0$ and $g$ restricted to $B \cap[(T-N) \times X]$ is Borel measurable.

There are at least two differences between our Theorem 4.1 and Wesley's theorem. First, Wesley's argument involves forcing techniques whereas our argument is purely descriptive set theoretic. Second, for each probability measure $\mu$, Wesley's construction yields a Borel set $N$ with $\mu(N)=0$ and a Borel measurable function $g$ from $B \cap[(T-N) \times X]$ into $R$ such that for each $t \in T-N, g(t, \cdot)$ is a continuous representation of $\preccurlyeq_{t}$. However, these $g$ 's may vary wildly with $\mu$. In our construction, we have a fixed $g$ and only vary the null set $N$ with $\mu$. Of course, our problem raised earlier asked whether it is even necessary to vary $N$ with $\mu$.

We will use the following conventions concerning a preference order $\leqslant: x \sim y$ means $x \preccurlyeq y$ and $y \preccurlyeq x ; x \prec y$ but not $y \preccurlyeq x$. Of course, if $\preccurlyeq$ is a continuous preference order, then each equivalence class is closed.

This paper grew out of and is an improvement upon an earlier unpublished manuscript of the author [11]. The central theme of that manuscript was to first give a number of different constructions of continuous representations of a fixed preference order (Debreu's theorem), and second, to "parametrize" these constructions in the setting of the fundamental hypothesis. The idea was that the more "effective" the construction the better the measurability properties of the parametrized function would be. It was shown that one could take a particular method of construction and obtain Wesley's theorem. This is also carried out in this paper. It was also shown that if one followed this procedure and if one assumed Martin's Axiom (MA), then a universally measurable parametrized representation could be obtained (actually, one could take any of the constructions given and obtain a universally measurable parametrized representation assuming MA). What this result suggested is that Martin's Axiom is not really necessary: there must be an absolute argument for such a representation. This is precisely what happened. This fact was discovered by at least three people who read the original manuscript, John Burgess [2,3] who used some techinques from "modern" descriptive set theory and by David Fremlin [8] and by the author both of whom used only "classical" descriptive set theoretic techniques (even though the key classical result used here, Lemma 3.2, has only been known for a few years). The proofs given in this paper follow the construction of Fremlin because this single method of construction shows most clearly how all the theorems may be obtained (although one could obtain similar results using any of the constructions given in [11]). 
We wish to thank the referee for his numerous corrections and suggestions concerning the original manuscript [11] and for pointing out that the map $g$ constructed in Theorem 3.4 is $\delta(T) \otimes \Re(X)$-measurable. We also wish to thank John Burgess for his comments and David Fremlin for his comments and permission to use his construction.

2. Basic construction of a continuous representation. Our proofs of the general theorems discussed in the introduction follow a basic strategy. First, one must have a method of proving the existence of a (continuous) representation of a given preference order. Then one "parametrizes" the proof. The idea being that if one is sufficiently clever in constructing a representation then the parametrized construction should retain some degree of measurability.

So, here we investigate representations of a fixed preference order. The first two theorems demonstrate a method of constructing a continuous representation of a given preference order. Theorem 2.2 is due to Debreu [5]. It is these proofs which will be parametrized in the next section.

THEOREM 2.1. Let $X$ be a second countable topological space and $\preccurlyeq$ a continuous preference order on $X$. Let $\left\{V_{n}\right\}_{n=1}^{\infty}$ be a base for the topology of $X$. For each $k$, let

$$
R_{k}=\left\{y: y \in V_{k} \text { and }\left[x \in V_{k} \rightarrow y \preccurlyeq x\right]\right\}
$$

and let

$$
L_{k}=\left\{y: y \in V_{k} \text { and }\left[x \in V_{k} \rightarrow x \preccurlyeq y\right]\right\} .
$$

Let $D=\left\{x_{n}: n \in N\right\}$ be a countable dense subset of $X$ such that for each $k$, if $R_{k} \neq \varnothing$, then $D \cap R_{k} \neq \varnothing$ and if $L_{k} \neq \varnothing$, then $D \cap L_{k} \neq \varnothing$. Define the function $h$ on $D$ as follows.

Set

$$
h\left(x_{1}\right)=1 / 2 .
$$

Suppose $h$ has been defined on $x_{1}, \ldots, x_{n}$. Then:

(a) If $\exists i \leqslant n\left[x_{i} \sim x_{n+1}\right]$, set $h\left(x_{n+1}\right)=h\left(x_{i}\right)$.

(b) If $x_{i} \prec x_{n+1}, \forall i \leqslant n$, set $h\left(x_{n+1}\right)=\frac{1}{2}\left(1+\max \left\{h\left(x_{i}\right): i \leqslant n\right\}\right)$.

(c) If $x_{n+1} \prec x_{i}, \forall i \leqslant n$, set $h\left(x_{n+1}\right)=\frac{1}{2}\left(\min \left\{h\left(x_{i}\right): x_{i} \leqslant n\right\}\right)$.

(d) If $x_{i_{0}} \prec x_{n+1} \prec x_{j_{0}}$ for some $i_{0}$ and $j_{0}, 1 \leqslant i_{0}, j_{0} \leqslant n$ and $\forall i \leqslant n, x_{i} \leqslant x_{i_{0}}$ or $x_{j_{0}} \preccurlyeq x_{i}$, set

$$
h\left(x_{n+1}\right)=\frac{1}{2}\left(h\left(x_{i_{0}}\right)+h\left(x_{j_{0}}\right)\right) .
$$

The function $h$ has the following properties:

(1) $x_{i} \preccurlyeq x_{j} \leftrightarrow h\left(x_{i}\right) \leqslant h\left(x_{j}\right)$, and

(2) if $\left\{x_{n_{i}}\right\}_{i=1}^{\infty}$ and $\left\{x_{m_{i}}\right\}_{i=1}^{\infty}$ are sequences of elements of $D$ such that

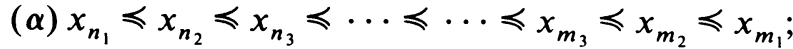

( $\beta$ ) either $\left\{x_{n_{i}}\right\}_{i=1}^{\infty}$ is strictly increasing or $\left\{x_{m_{i}}\right\}_{i=1}^{\infty}$ is strictly decreasing; and

( $\gamma) \forall j \exists p\left[x_{j} \preccurlyeq x_{n_{p}}\right.$ or $\left.x_{m_{p}} \preccurlyeq x_{j}\right]$,

then $\lim _{i \rightarrow \infty} h\left(x_{n_{i}}\right)=\lim _{i \rightarrow \infty} h\left(x_{m_{i}}\right)$. 
Proof. It can be checked that $h$ satisfies condition (1). Assume the hypotheses of condition (2) hold and

$$
\lim _{i \rightarrow \infty} h\left(x_{n_{i}}\right)=b-\varepsilon<b+\varepsilon=\lim _{i \rightarrow \infty} h\left(x_{m_{i}}\right) .
$$

Notice that condition $(\gamma)$ guarantees us that for all $k$, either $h\left(x_{k}\right) \leqslant b-\varepsilon$ or $b+\varepsilon \leqslant h\left(x_{k}\right)$. Let $i$ and $j$ be the first integers such that $b-2 \varepsilon<h\left(x_{i}\right) \leqslant b-\varepsilon$ and $b+\varepsilon \leqslant h\left(x_{j}\right)<b+2 \varepsilon$. Let us suppose that $i<j$. Let $s$ be an integer less than $j$ such that $b-2 \varepsilon<h\left(x_{s}\right) \leqslant b-\varepsilon$ and if $k \leqslant j$ and $h\left(x_{k}\right) \leqslant b-\varepsilon$, then $x_{k} \leqslant x_{s}$. So, if $k \leqslant j$, either $x_{k} \leqslant x_{s}$ or $x_{j} \leqslant x_{k}$. Condition $(\gamma)$ guarantees us that there is a first positive integer $p$ such that $x_{s} \prec x_{p} \prec x_{j}$. Of course, $p>j$. Now, by part (d) of the definition of $h, h\left(x_{p}\right)=\frac{1}{2}\left(h\left(x_{s}\right)+h\left(x_{j}\right)\right)$. But, $\left|h\left(x_{p}\right)-b\right|<\varepsilon$. This contradiction establishes part (2) of the theorem in case $i<j$. The argument in case $j<i$ is similar. Q.E.D.

THEOREM 2.2. Let $X$ be a second countable topological space, $\preccurlyeq a$ continuous preference order on $X$ and let $D$ and $h$ be as constructed in Theorem 2.1. Define the function $g$ from $X$ into $[0,1]$ by setting

$$
g(x)=\inf \left\{h\left(x_{i}\right): x \preccurlyeq x_{i}\right\} .
$$

Then $g$ is a continuous representation of $\preccurlyeq$.

Proof. Certainly, if $x \leqslant y$, then $g(x) \leqslant g(y)$ and $g \mid D=h$.

Suppose $g(x) \leqslant g(y)$ and $y \prec x$. If there were elements $x_{j}$ and $x_{i}$ of $D$ such that $y \leqslant x_{i} \prec x_{j} \leqslant x$, then $g(y) \leqslant g\left(x_{i}\right)=h\left(x_{i}\right)<h\left(x_{j}\right)=g\left(x_{j}\right) \leqslant g(x)$. From this contradiction it follows that there is an open set $U$ such that $y \in U$ and if $z \in U$, then $z \preccurlyeq y$. Thus, there is some $k_{1}$ such that $y \in R_{k_{1}}$. Therefore, there is some $n_{1}$ such that $y \sim x_{n_{1}}$. Similarly, there is $k_{2}$ such that $x \in L_{k_{2}}$ and some $n_{2}$ such that $x \sim x_{n_{2}}$. But, this implies that $g(y) \prec g(x)$. This last contradiction leads to the conclusion that if $g(x) \leqslant g(y)$, then $x \leqslant y$.

In order to prove that $g$ is continuous, we will demonstrate that $g$ is left continuous at some fixed point $y$. A similar argument can be given to show that $g$ is right continuous at $y$, and of course, $g$ is continuous at $y$ if and only if $g$ is both right and left continuous at $y$. So, let $\left\{y_{n}\right\}_{n=1}^{\infty}$ be a sequence of elements of $X$ converging to $y$ such that $y_{1} \preccurlyeq y_{2} \preccurlyeq y_{3} \preccurlyeq \cdots$ and suppose $\lim _{n \rightarrow \infty} g\left(y_{n}\right)=l<g(y)$. Notice that for each $n, y_{n} \prec y$ and the sequence $\left\{y_{n}\right\}_{n=1}^{\infty}$ must have a strictly increasing subsequence. From this fact, one can construct a sequence $\left\{x_{n_{i}}\right\}_{i=1}^{\infty}$ of elements of $D$ which is strictly increasing such that $\left\{x_{n_{i}}\right\}$ converges to $y$ and $\lim _{i \rightarrow \infty} g\left(x_{n_{i}}\right)=$ $\lim _{i \rightarrow \infty} h\left(x_{n_{i}}\right)=l$. Now, if there is some element $x_{j}$ of $D$ such that $x_{j} \sim y$, let $x_{m_{i}}=x_{j}$, for $i=1,2,3, \ldots$ Otherwise, let $\left\{x_{m_{i}}\right\}_{i=1}^{\infty}$ be a decreasing sequence of elements of $D$ such that $g(y)=\lim _{i \rightarrow \infty} h\left(x_{m_{i}}\right)$. The sequences $\left\{x_{n_{i}}\right\}$ and $\left\{x_{m_{i}}\right\}$ satisfy the hypotheses of (2) in Theorem 2.1, but violate the conclusion of (2). This contradiction demonstrates that $g$ is left continuous at $y$. Q.E.D.

3. Parametrized constructions. In this section, we shall parametrize the constructions of $\S 2$. We shall assume the fundamental hypothesis. In addition, let $\left\{V_{n}\right\}_{n=1}^{\infty}$ be a base for the topology of $X$ and for each $k$, let

$$
R_{k}=\left\{(t, y): y \in V_{k} \cap B_{t} \text { and }\left[x \in V_{k} \cap B_{t} \rightarrow y \preccurlyeq_{t} x\right]\right\},
$$


and let

$$
L_{k}=\left\{(t, y): y \in V_{k} \cap B_{t} \text { and }\left[x \in V_{k} \cap B_{t} \rightarrow x \preccurlyeq_{t} y\right]\right\} .
$$

Let $\mathcal{C}$ be the family of all subsets of $T \times X$ which can be obtained from operation (A) applied to sets of the form $C \times D$, where $C \in \delta(T)$ and $D \in \mathscr{B}(X)$. Here $S(Z)$ denotes the $C$-sets of Selivanovskii, the smallest family of subsets of $Z$ containing the open sets which is closed under complementation and operation (A) [1].

LEMMA 3.1. Each of the sets $R_{k}$ and $L_{k}$ is in $\mathcal{C}$.

Proof. We have

$$
(t, y) R_{k} \leftrightarrow(t, y) \in\left(T \times V_{k}\right) \cap B
$$

and (if $V_{j} \subseteq V_{k}$ and $V_{j} \cap B_{t} \neq \varnothing$, then $\exists z\left[z \in V_{j} \cap B_{t}\right.$ and $\left.y \preccurlyeq_{t} z\right]$ ). Thus,

$$
\begin{aligned}
R_{k}= & B \cap\left(T \times V_{k}\right) \\
\cap & \bigcap_{V_{j} \subseteq V_{k}}\left[\left(\left(T-\pi_{12}\left(\left(T \times V_{j}\right) \cap B\right)\right) \times X\right) \cup \pi_{12}\left(E \cap\left(T \times X \times V_{j}\right)\right)\right] .
\end{aligned}
$$

Q.E.D.

LEMMA 3.2. If $W \in \mathcal{C}$, then there is an $\delta$-measurable map from $T$ into $X$ such that if $W_{t} \neq \varnothing$, then $f(t) \in W_{t}$.

A proof of this theorem has been given by Dellacherie [6, p. 217]. This theorem seems to have been known to a number of people.

The next theorem is a parametrized version of Theorem 2.1.

TheOREM 3.3. Assume the fundamental hypothesis. There is a sequence $\left\{f_{n}\right\}_{n=1}^{\infty}$ of maps of $T$ into $X$ such that (1) for each $n, f_{n}$ is $\delta(T)$-measurable, (2) for each $t$, $D_{t}=\left\{f_{n}(t): n \in N\right\}$ is a dense subset of $B_{t}$ and (3) if $R_{k t} \neq \varnothing$, then $D_{t} \cap R_{k t} \neq \varnothing$ and if $L_{k t} \neq \varnothing$, then $D_{t} \cap L_{k t} \neq \varnothing$.

Proof. Since $B$ is an analytic subset of $T \times X$, it follows from the von NeumannYankov theorem (see [15]) that there is a sequence $\left\{g_{n}\right\}_{n=1}^{\infty}$ of maps of $T$ into $X$ each of which is $\mathscr{B Q}(T)$-measurable and such that for each $t,\left\{g_{n}(t): n \in N\right\}$ is dense in $B_{t}$.

For each $k$, let $h_{k}$ be an $\delta(T)$-measurable map of $T$ into $X$ such that if $R_{k t} \neq \varnothing$, then $h_{k}(t) \in R_{k t}$. Let $H_{k}=\left\{t:\left(t, h_{k}(t)\right) \in R_{k}\right\}$. Then $H_{k} \in \mathcal{S}(T)$. Let $\hat{h}_{k}(t)=h_{k}(t)$, if $t \in H_{k}$ and let $\hat{h}_{k}(t)=g_{1}(t)$, if $t \notin H_{k}$. Then $\hat{h}_{k}$ is an $\delta(T)$-measurable map of $T$ into $X$ and for each $t, \hat{h}_{k}(t) \in B_{t}$ and if $R_{k t} \neq \varnothing, \hat{h}_{k}(t) \in R_{k t}$. For each $k$, one can construct a similar map $p_{k}$ for $L_{k}$. Finally, arranging the sequences $\left\{g_{k}\right\},\left\{\hat{h}_{k}\right\}$, and $\left\{p_{k}\right\}$ into one sequence $\left\{f_{k}\right\}_{n=1}^{\infty}$, we obtain the conclusion of the theorem.

THEOREM 3.4. Assume the fundamental hypothesis. Then there is a map $g$ of $B$ into $R$ which is $\mathcal{S}(T) \otimes \mathscr{B}(X)$-measurable and such that for each $t, g(t, \cdot)$ is a continuous representation of $\preccurlyeq_{t}$.

Proof. Let $\left\{f_{n}\right\}_{n=1}^{\infty}$ be a sequence of $\delta(T)$-measurable maps satisfying the conclusion of Theorem 3.3. Let $D=\bigcup\left\{\operatorname{Gr}\left(f_{n}\right): n=1,2,3, \ldots\right\}$ and define $h$ on $D$ 
by the following procedure. First, let $h\left(t, f_{1}(t)\right)=\frac{1}{2}$, for all $t$ in $T$. Certainly, $h$ is $\delta(T) \otimes \mathscr{B}(X)$-measurable since $h^{-1}(E)$ is either empty or is $\operatorname{Gr}\left(f_{1}\right)$ which is in $\delta(T) \otimes \Re(X)$. Next, let

and

$$
\begin{aligned}
& T_{1}=\left\{t: f_{2}(t) \prec_{t} f_{1}(t)\right\}, \\
& T_{2}=\left\{t: f_{1}(t) \sim_{t} f_{2}(t)\right\},
\end{aligned}
$$

$$
T_{3}=\left\{t: f_{1}(t) \prec_{t} f_{2}(t)\right\} .
$$

Each of the sets $T_{1}, T_{2}$, and $T_{3}$ is in $\delta(T)$. Now, extend the domain of $h$ to include $\operatorname{Gr}\left(f_{2}\right)$ by setting

$$
h\left(t, f_{2}(t)\right)= \begin{cases}\frac{1}{4}, & \text { if } t \in T_{1}, \\ \frac{1}{2}, & \text { if } t \in T_{2}, \\ \frac{1}{3}, & \text { if } t \in T_{3} .\end{cases}
$$

Now, continue this process by induction as in Theorem 2.1. It can be checked that the map $h$ is $\delta(T) \otimes \mathscr{B}(X)$-measurable. Finally, define $g$ on $B$ by setting

$$
g(t, x)=\inf \left\{h\left(\left(t, f_{n}(t)\right)\right): x \preccurlyeq_{t} f_{n}(t)\right\} .
$$

Since $g(t, \cdot)$ is the parametrized version of the function constructed in Theorem 2.2, $g(t, \cdot)$ is a continuous representation of $\preccurlyeq_{t}$ for each $t$ in $T$.

In order to see that $g$ is $\delta(T) \otimes \Re(X)$-measurable, fix $a$ and let $V=g^{-1}((-\infty, a))$. Note that

$$
V=\left\{(t, x): \exists n\left[x \preccurlyeq_{t} f_{n}(t) \text { and } h\left(t, f_{n}(t)\right)<a\right\}\right.
$$

Thus,

$$
V=\bigcup_{n}\left[\left\{(t, x): x \preccurlyeq_{t} f_{n}(t)\right\} \cap\left\{t: h\left(t, f_{n}(t)\right)<a\right\} \times X\right] .
$$

For each $n$, let $F_{n}(t, x)=\left(t, x, f_{n}(t)\right)$. Then $F_{n}$ is an $\mathcal{S}(T) \otimes \mathscr{B}(X)$-measurable map of $B$ into $T \times X \times X$. So,

$$
\left\{(t, x): x \preccurlyeq_{t} f_{n}(t)\right\}=F_{n}^{-1}(E)
$$

and is therefore in $\delta(T) \otimes \mathscr{B}(X)$. Also, for each $n$, the map $t \mapsto\left(t, f_{n}(t)\right)$ is an $\delta(T)$-measurable map of $T$ into $D$ and since $t \mapsto h\left(t, f_{n}(t)\right)$ is the composition of two $\delta$-measurable maps, it is $\delta(T)$-measurable [14; 4,p. 403]. This implies $\left\{t: h\left(t, f_{n}(t)\right)\right.$ $<a\}$ is in $\delta(T)$. Therefore, $g$ is $\delta(T) \otimes \mathscr{B}(X)$-measurable. Q.E.D.

4. Applications and Borel measurable selections of continuous representations. In this section we apply the techniques of $\S 3$ to obtain Wesley's theorem and to obtain some sufficient conditions for the existence of a Borel measurable selection of continuous representations. First, we give an improvement of Wesley's theorem [16].

Theorem 4.1. Assume the fundamental hypothesis. There is a map $g$ of $B$ into $R$ which is $\delta(T) \otimes \mathscr{B}(X)$-measurable such that for each $t, g(t, \cdot)$ is a continuous representation of $\preccurlyeq_{t}$ and such that if $\mu$ is a probability measure defined on the Borel subsets of $T$, then there is a Borel set $N$ such that $\mu(N)=0$ and $g$ restricted to $B \cap[(T-N) \times X]$ is Borel measurable. 
Proof. Let $\left\{f_{n}\right\}_{n=1}^{\infty}$ be a sequence of $\delta(T)$-measurable maps as constructed in Theorem 3.3. Since these maps are universally measurable there is a Borel subset $N$ of $T$ with $\mu(N)=0$ such that for each $n, f_{n} \mid T^{\prime}$ is Borel measurable where $T^{\prime}=T-N$. Now, note that our constructions of $h$ and $g$ in Theorems 3.4 from the maps $f_{n}$ will be Borel measurable maps. Q.E.D.

In fact, the preceding argument can be used (with $N=\varnothing$ ) to prove the following theorem.

Theorem 4.2. Assume the fundamental hypothesis. Suppose there is a sequence $\left\{f_{n}\right\}_{n=1}^{\infty}$ of Borel measurable maps of $T$ into $X$ such that conditions (2) and (3) of Theorem 3.3 hold. Then there is a Borel measurable map $g$ of $B$ into $[0,1]$ such that for each $t, g(t, \cdot)$ is a continuous representation of $\preccurlyeq_{t}$.

THEOREM 4.3. Assume the fundamental hypothesis. Furthermore assume that for each $t, B_{t}$ is $\sigma$-compact. Then there is a Borel measurable $g$ from $B$ into $[0,1]$ such that for each $t, g(t, \cdot)$ is a continuous representation of $\preccurlyeq_{t}$.

Proof. First, notice that there is a sequence $\left\{d_{n}: n \in N\right\}$ of Borel measurable maps of $T$ into $X$ such that for each $t,\left\{d_{n}(t): n \in N\right\}$ is a dense subset of $B_{t}$ [13]. Next, notice that for each $k, R_{k t}$ is closed with respect to $B_{t}$. So, each $R_{k t}$ is $\sigma$-compact. Also, each set $R_{k}$ is a Borel set. This follows from the expression for $R_{k}$ given in (3.1) and the facts that if $V$ is open in $X$, then $(T \times X) \cap B$ is a Borel set with each $T$-section $\sigma$-compact and $W=E \cap(T \times X \times V)$ is a Borel set in $T \times X$ $\times X$ such that for each $(t, x) \in T \times X, W_{(t, x)}=\{z:(t, x, z) \in W\}$ is $\sigma$-compact. Thus, the projections of these sets are Borel sets [8] and therefore $R_{k}$ is a Borel set. Thus, for each $k$, there is a Borel measurable map $h_{k}$ of $T$ into $X$ such that for each $t$, $h_{k}(t) \in B_{t}$ and if $R_{k t} \neq \varnothing$, then $h_{k}(t) \in R_{k t}$. Similarly, one can construct Borel maps for the sets $L_{k}$. Thus, there is a sequence $\left\{f_{n}\right\}_{n=1}^{\infty}$ of Borel measurable maps of $T$ into $X$ satisfying properties (2) and (3) of Theorem 3.3. The corollary now follows. Q.E.D.

Corollary 4.4. Assume the fundamental hypothesis. Furthermore, assume that for each $t, \preccurlyeq_{t}$ is the linear order and assume that there is a sequence $\left\{g_{n}\right\}_{n=1}^{\infty}$ of Borel measurable maps of $T$ into $X$ such that for each $t,\left\{g_{n}(t): n \in N\right\}$ is a dense subset of $B_{t}$. Then there is a Borel measurable map $g$ of $B$ into $[0,1]$ such that for each $t, g(t, \cdot)$ is a continuous representation of $\preccurlyeq_{t}$.

Proof. All that needs to be shown is that each of sets $R_{k}\left(L_{k}\right)$ is a Borel set, since for each $t, R_{k t}\left(L_{k t}\right)$ is $\sigma$-compact being either empty or consisting of a single point.

But,

$$
(t, y) \in R_{k} \leftrightarrow y \in V_{k} \cap B_{t} \text { and } \forall n\left[g_{n}(t) \preccurlyeq_{t} y \text { or } g_{n}(t) \notin V_{k}\right] .
$$

This means each set $R_{k}$ (and similarly each $L_{k}$ ) is a Borel set. Q.E.D.

In closing we would like to raise two questions.

Question 1. Assume the fundamental hypothesis. Is there a map $f: B \rightarrow R$ which is $\mathscr{G} Q(T) \otimes \mathscr{B}(X)$-measurable and for each $t, f(t, \cdot)$ is a continuous representation of $\preccurlyeq$ ? 
Question 2. Assume the fundamental hypothesis and assume that for each $t, \preccurlyeq$, is a pre-wellordering of $B_{t}$. Is there a Borel measurable map $f: B \rightarrow R$ such that for each $t, f(t, \cdot)$ is a continuous representation of $\preccurlyeq_{t}$ ?

\section{REFERENCES}

1. J. P. Burgess, Classical hierarchies from a modern standpoint, Part I. C-sets, Fund. Math. (to appear).

2. Personal communication, 1981.

3. From preference to utility, a problem of descriptive set theory, preprint.

4. D. Cenzer and R. D. Mauldin, Measurable parametrizations and selections, Trans. Amer. Math. Soc. 245 (1978), 399-408.

5. G. Debreu, Continuity properties of Paretian utility, Internat. Econom. Rev. 5 (1964), 285-293.

6. C. Dellacherie, Un cours sur les ensembles analytiques, Analytic Sets, edited by C. A. Rogers et al., Academic Press, New York, 1980.

7. A. M. Faden, Economics of space and time, The measure-theoretic foundations of social science, Iowa State Univ. Press, Ames, Iowa, 1977

8. D. Fremlin, Personal communication, 1981.

9. K. Kuratowski, Topology, Vol. I, Academic Press, New York, 1966.

10. R. D. Mauldin, The boundedness of the Cantor-Bendixson order of some analytic sets, Pacific J. Math. 74 (1978), 167-177.

11. Measurable constructions of preference orders, unpublished manuscript.

12. J. T. Rader, The existence of a utility function to represent preferences, Rev. Econom. Stud. 30 (1963), 229-232.

13. J. Saint-Raymond, Boréliens à coupes $K_{\sigma}$, Bull. Soc. Math. France 104 (1976), 389-400.

14. S. E. Shreve, Probability measures and the C-sets of Selivanovskii, Pacific J. Math. 79 (1978), 189-196.

15. D. Wagner, Survey of measurable selection theorems, SIAM J. Control Optimization 15 (1977), 859-903.

16. E. Wesley, Borel preference orders in markets with a continuum of traders, J. Math. Econom. 3 (1976), 155-165. MR \#11955

Department of Mathematics, North Texas State University, Denton, TeXas 76203 